\title{
Some remarks on the Einstein and Møller pseudotensors for static and spherically-symmetric configurations
}

\author{
Jerzy Matyjasek* \\ Institute of Physics, Maria Curie-Sktodowska University, \\ pl. Marii Curie-Sktodowskiej 1, 20-031 Lublin, Poland
}

\begin{abstract}
It is shown that for the spherically-symmetric and static systems the hypotheses posed by Yang and Radinschi and by Vagenas can be related to the particular distribution of the source. Simple proofs are given and a number of examples are discussed with the special emphasis put on the quantum corrected Schwarzschild black hole.
\end{abstract}

Recent interest in the pseudotensors describing the energy-momentum of gravity has been stimulated to a large extent by the understanding that the freedom in defining the pseudotensors is associated with the freedom in the choice of the Hamiltonian boundary terms [1. Thus, each pseudotensor reflects particular physical situation, and, being in fact quasilocal [2, is acceptable. This removed (at least partially) the anathema pronounced by a number of authors (see for example Ref. 3) and caused that the energy momentum pseudotensors returned from the exile and have settled themselves in the main stream of the theory. There are quite a number of various constructions, each of noble parentage: Einstein 4], Landau-Lifshitz [5, Weinberg [6], Møller 7], Bergman [8] and Papapetrou 9], to name a few. Each has been a subject of detailed studies and a source of interesting and important results. The subjects covered range form the black hole physics to gravitational waves. (See, for example, Refs. 10-18 and references cited therein). Despite extensive work done so far there is still room for new findings and fresh insights.

Some time ago Radinschi and Yang [17] observed that for the Reissner-Nordström and the regular black holes [19, 20, 21, 22, the difference between the energy calculated with the aid of the Einstein pseudotensor, $E_{E}$, and this obtained employing the Møller prescription, $E_{M}$, can be related to the energy density, $\rho$, of the matter fields

$$
E_{E}-E_{M} \sim r^{3} \rho .
$$

Later Vagenas [18] found that for the Reissner-Nordström (RN) and the RN dyadosphere 23 there is an interesting relation between the coefficients of the expansion of $E_{E}$ and $E_{M}$ in the inverse powers of $r$. This has been partially confirmed by the behaviour of a few first coefficients of the expansion of the regular black hole solution. He hypothesized that if $\alpha_{k}$ and $\beta_{k}$ are, respectively, the expansion coefficients of $E_{E}$ and $E_{M}$, then

$$
\alpha_{k}=\frac{1}{k+1} \beta_{k}
$$

\footnotetext{
*email: jurek@kft.umcs.lublin.pl, matyjase@tytan.umcs.lublin.pl
} 
It is the aim of this letter to demonstrate how these observations and hypotheses can be proven and understood in relation with the source term of the Einstein field equations, (i. e., the stress-energy tensor of the matter fields) and to analyse their limitations. The discussion will be illustrated with a few examples. As our demonstration heavily relies on the particular representation of the line element describing static and spherically-symmetric configuration we shall start with a few basic facts.

Let us consider the general spherically-symmetric, static and asymptotically flat geometry described by the line element (our conventions follow these of MTW)

$$
d s^{2}=-e^{2 \psi(r)}\left(1-\frac{2 M(r)}{r}\right) d t^{2}+\left(1-\frac{2 M(r)}{r}\right)^{-1} d r^{2}+r^{2}\left(d \theta^{2}+\sin ^{2} \theta d \phi^{2}\right),
$$

where $M(r)$ and $\psi(r)$ are two functions that are to be determined from the Einstein field equations, supplemented by the physically motivated boundary conditions. This representation of the line element has proven to be useful in various contexts as the Einstein field equations have particularly transparent form 24. Moreover, let us assume that the (total) covariantly conserved stress-energy tensor of the material fields can be written in the form dictated by the symmetries of the problem

$$
T_{\nu}^{\mu}=\operatorname{diag}[a(r), b(r), c(r), c(r)] .
$$

The $\left(\begin{array}{l}0 \\ 0\end{array}\right)$ component of the Einstein equations reads

$$
-\frac{2}{r^{2}} \frac{d M(r)}{d r}=8 \pi a(r)
$$

whereas their $\left(\begin{array}{l}1 \\ 1\end{array}\right)$ component can be written as

$$
-\frac{2}{r^{2}} \frac{d M(r)}{d r}+\frac{2}{r}\left(1-\frac{2 M(r)}{r}\right) \psi^{\prime}(r)=8 \pi b(r) .
$$

In the latter we shall employ the boundary conditions in the form

$$
M(\infty)=\mathcal{M}, \quad \psi(\infty)=0,
$$

where $\mathcal{M}$ is the total mass of the system as seen by a distant observer. On the other hand, for the black hole configurations, one can also take

$$
M\left(r_{+}\right)=\frac{r_{+}}{2}, \quad \psi(\infty)=0,
$$

where $r_{+}$is the location of the event horizon, defined here as the outermost trapped surface. From (5) one has

$$
\frac{d M(r)}{d r}=-4 \pi r^{2} a(r)
$$

whereas from (5) and (6) one obtains

$$
\frac{d \psi(r)}{d r}=4 \pi r \frac{b(r)-a(r)}{1-\frac{2 M(r)}{r}}
$$

Now, let us return to the general form of the line element and calculate the Einstein pseudotensor [4. The Einstein pseudotensor, $\Theta_{\lambda}^{\mu}$, can easily be constructed in the Cartesiantype coordinates by differentiation of the Freud superpotential, $V_{\lambda}^{\mu \nu}$,

$$
\Theta_{\lambda}^{\mu}=\frac{1}{16 \pi} \frac{\partial}{\partial x^{\nu}} V_{\lambda}^{\mu \nu},
$$


where

$$
V_{\lambda}^{\mu \nu}=\frac{1}{\sqrt{-g}} g_{\lambda \tau} \frac{\partial}{\partial x^{\gamma}}\left[g\left(g^{\mu \tau} g^{\nu \gamma}-g^{\nu \tau} g^{\mu \gamma}\right)\right] .
$$

The $\Theta_{\lambda}^{\mu}$ does not exhaust the list of interesting and important pseudotensors. Indeed, there are half a dozen other objects (sometime referred to as complexes) that are useful in various circumstances. One of the most studied, however, is the pseudotensor constructed by Møller in the late fifties of the last century. One of the advantages of the Møller pseudotensor, $\mathcal{T}_{\nu}^{\mu}$, is that it can be calculated in any coordinate system. Its construction proceeds in two steps: first, one has to calculate the superpotential

$$
U_{\nu}^{\mu \lambda}=-\sqrt{-g}\left(\frac{\partial}{\partial x^{\kappa}} g_{\nu \sigma}-\frac{\partial}{\partial x^{\sigma}} g_{\nu \kappa}\right) g^{\mu \kappa} g^{\lambda \sigma},
$$

and, subsequently, the energy-momentum pseudotensor

$$
\mathcal{T}_{\nu}^{\mu}=\frac{1}{8 \pi} \frac{\partial}{\partial x^{\lambda}} U_{\nu}^{\mu \lambda} .
$$

Due to the antisymmetry of the superpotentials the pseudotensors (11) and (14) are divergence-free. The energy and momentum are given by

$$
P_{i}=\iiint \tau_{i}^{0} d x^{1} d x^{2} d x^{3}
$$

and

$$
E=\iiint \tau_{0}^{0} d x^{1} d x^{2} d x^{3},
$$

respectively, where $\tau_{\nu}^{\mu}$ stands either for $\Theta_{\nu}^{\mu}$ or $\mathcal{T}_{\nu}^{\mu}$. In the latter we shall differentiate between them simply by inserting subscripts $E$ or $M$.

The energy calculated with the aid of the Møller energy-momentum pseudotensor for the geometry (3) assumes the simple form

$$
E_{M}=e^{\psi(r)}\left[M(r)-r M^{\prime}(r)+r^{2} \psi^{\prime}(r)-2 r M(r) \psi^{\prime}(r)\right] .
$$

On the other hand, the calculations of the Einstein pseudotensor is slightly more involved. Indeed, as have been said earlier one has to introduce the standard quasi-Cartesian coordinates $\{x, y, z\}$ and rewrite the line element (3) in the form

$$
\begin{aligned}
d s^{2} & =-e^{2 \psi(r)}\left(1-\frac{2 M(r)}{r}\right) d t^{2}+\left(d x^{2}+d y^{2}+d z^{2}\right) \\
& +\frac{1}{r^{2}}\left[\left(1-\frac{2 M(r)}{r}\right)^{-1}-1\right](x d x+y d y+z d z)^{2} .
\end{aligned}
$$

From the definition of the Einstein pseudotensor, after some algebra, one obtains remarkably simple result

$$
E_{E}=e^{\psi(r)} M(r) .
$$

The calculations have been carried out with the aid of Maxima package, and in the advent of the computer algebra systems, this problem should be regarded as a five-finger exercise. Identical results can easily be obtained from the formulas constructed by Virbhadra 25] and Xulu [26] for the Einstein and Møller pseudotensors, respectively, in the most general nonstatic and spherically-symmetric geometry.

Now, let us assume that $a(r)$ falls sufficiently rapidly as $r \rightarrow \infty$ to make the integral (9) finite and $a(r)=b(r)$. Making use of the boundary conditions (7) one has $\psi(r)=0$ and

$$
M(r)=\mathcal{M}+4 \pi \int_{\infty}^{r} r^{2} a(r) d r=\mathcal{M}+M_{0}(r) .
$$


On the other hand, the boundary conditions (8) yield

$$
M(r)=\frac{r_{+}}{2}+4 \pi \int_{r_{+}}^{r} r^{2} a(r) d r=\frac{r_{+}}{2}+\tilde{M}_{0}(r) .
$$

For the simplified configuration the Møller energy reads

$$
E_{M}=M(r)-r M^{\prime}(r),
$$

whereas the Einstein energy coincides with the function $M(r)$ as can easily be seen from Eq.(19):

$$
E_{E}=M(r) .
$$

Now, we are ready to demonstrate a simple relation existing between the expansions (in terms of $r^{-1}$ ) of the Einstein and Møller energy. Following Vagenas, let us assume that

$$
M_{0}(r)=\sum_{k=1}^{\infty} \alpha_{k} r^{-k}
$$

where $\alpha_{k}$ are numerical coefficients. It should be noted that there are quite a number of solutions that belong to that class. Indeed, the Reissner-Nordström, the regular black holes or the geometry of the dyadosphere of RN black hole may serve as the simple examples of particular realizations of (24) with

$$
\begin{gathered}
\alpha_{1}=-\frac{Q^{2}}{2}, \quad \alpha_{k}=0 \quad \text { for } \quad k \geq 2, \\
\alpha_{k}=\frac{1}{k !} \frac{d^{k}}{d \xi^{k}} M_{0}(\xi)_{\mid \xi=0,}, \quad \xi=\frac{1}{r},
\end{gathered}
$$

and

$$
\alpha_{1}=-\frac{Q^{2}}{2}, \quad a_{2}=\alpha_{3}=\alpha_{4}=0, \quad \alpha_{5}=\sigma \frac{Q^{4}}{10}, \quad \alpha_{k}=0 \quad \text { for } k \geq 6,
$$

respectively. We do not attempt, of course, to list all the solutions of this type here.

It can easily be demonstrated that if $M_{0}(r)$ is given by (24) then

$$
E_{E}=\mathcal{M}+\sum_{k=1}^{\infty} \alpha_{k} r^{-k}
$$

and

$$
E_{M}=\mathcal{M}+\sum_{k=1}^{\infty}(k+1) \alpha_{k} r^{-k} \equiv \mathcal{M}+\sum_{k=1}^{\infty} \beta_{k} r^{-k}
$$

and, consequently,

$$
\alpha_{k}=\frac{1}{k+1} \beta_{k} .
$$

The above equation is precisely the relations found by Vagenas [18. He correctly hypothesized that it should have a wider domain of applicability than two or three special cases considered in Ref. 18. It should be emphasized, however, that in this demonstration the crucial role is played by the special form of the stress-energy tensor.

On the other hand, still working with the simplified stress-energy tensors of the matter fields one has

$$
\begin{aligned}
\Delta E & =E_{M}-E_{E}=-r M^{\prime}(r) \\
& =4 \pi r^{3} a(r)=4 \pi r^{3} T_{0}^{0},
\end{aligned}
$$


where we have used Eq. (9). This result for particular configurations has been obtained by Yang and Radinschi in Ref. 17. Finally observe, that for $a(r) \neq b(r)$ the relations (30) and (31) do not hold in general.

Equipped with the results (22) and (23) let us consider a slightly more complicated example of the quantum corrected black hole being a solution of the semiclassical Einstein field equations. The idea that lies behind the calculations is simple. One has to construct the stress-energy tensor for a general background (3) and subsequently solve (self-consistently) the differential equations with the boundary conditions (7) or (8).

The stress-energy tensor of the quantized massive scalar, spinor and vector fields can be obtained from the one-loop renormalized effective action, $W_{R}$, in a standard way, i.e., by a functional differentiation of $W_{R}$ with respect to the metric tensor. It gives rise to the quantum-corrected black hole described by the line element (3). The most general expression describing the approximate renormalized stress-energy tensor of the quantized massive scalar, spinor and vector fields have been constructed in Refs. 27 and 28. These formulas generalize the earlier results obtained by Frolov and Zel'nikov [29, 30, 31] (valid for the Ricci-flat geometries) and those of Ref. 32, where the stress-energy tensor has been calculated for the massive scalar fields in the general static, spherically-symmetric geometries.

In what follows we shall employ the boundary conditions (7), i.e., we shall parametrize the solution by the total mass of the system (consisitng of both classical and quantum parts) rather than the bare black hole mass. The resulting differential equations are, in general, too complicated to be solved exactly and consequently one has to refer to approximations. Fortunately, one can easily adopt an approach in which the stress-energy tensor of the quantized fields can be regarded as a small perturbation. Indeed, this can be done simply because the total stress-energy tensor of the matter fields can be written as

$$
T_{\nu}^{\mu}=\operatorname{diag}\left[a(r)+\varepsilon\left\langle T_{t}^{t}\right\rangle_{\text {ren }}^{(s)}, b(r)+\varepsilon\left\langle T_{r}^{r}\right\rangle_{\text {ren }}^{(s)}, c(r)+\varepsilon\left\langle T_{\theta}^{\theta}\right\rangle_{\text {ren }}^{(s)}, c(r)+\varepsilon\left\langle T_{\phi}^{\phi}\right\rangle_{\text {ren }}^{(s)}\right],
$$

where $\left\langle T_{\nu}^{\mu}\right\rangle_{\text {ren }}^{(s)}$ is the approximate renormalized stress-energy tensor of the quantized massive scalar $(s=0)$, spinor $(s=1 / 2)$ and vector $(s=1)$ fields, and $\varepsilon$ is the auxiliary parameter that helps to keep track of various terms in complicated expansions. (One should put $\varepsilon=1$ in a final stage of calculations).

To simplify discussion let us assume that for the classical sources the condition $a(r)=b(r)$ holds. This allows us to represent the functions $M(r)$ and $\psi(r)$, with a small abuse of notation, in the form 1

$$
M(r)=M_{0}(r)+\varepsilon M_{1}(r)+O\left(\varepsilon^{2}\right) \quad \text { and } \quad \psi(r)=\varepsilon \psi_{1}(r)+O\left(\varepsilon^{2}\right) .
$$

Now, restricting to the first-order perturbations one has the following system of the differential equations for $M_{1}(r)$ and $\psi_{1}(r)$ :

$$
\frac{d M_{1}(r)}{d r}=-4 \pi r^{2}\left\langle T_{t}^{t}\right\rangle_{r e n}^{(s)}
$$

and

$$
\frac{d \psi_{1}(r)}{d r}=4 \pi r \frac{\left\langle T_{r}^{r}\right\rangle_{r e n}^{(s)}-\left\langle T_{t}^{t}\right\rangle_{r e n}^{(s)}}{1-\frac{2 M_{0}(r)}{r}} .
$$

The energy calculated from the Einstein pseudotensor is

$$
E_{E}=M_{0}(r)+\left[M_{0}(r) \psi_{1}(r)+M_{1}^{\prime}(r)\right] \varepsilon+O\left(\varepsilon^{2}\right)
$$

\footnotetext{
${ }^{1}$ The function $M_{0}(r)$ in Eq. 33 must not be confused with the one defined in Eq. 20
} 
whereas the Møller energy is given by

$$
\begin{aligned}
E_{M} & =M_{0}(r)-r M_{0}^{\prime}(r)+\left[M_{0}(r) \psi_{1}(r)+M_{1}^{\prime}(r)-r \psi_{1}(r) M_{0}^{\prime}\right. \\
& \left.-r M_{1}^{\prime}(r)+r^{2} \psi_{1}^{\prime}(r)-2 r M_{0}(r) \psi_{1}^{\prime}(r)\right] \varepsilon+O\left(\varepsilon^{2}\right)
\end{aligned}
$$

and the difference $\Delta E$ is

$$
\Delta E=-r M_{0}^{\prime}(r)-\left[\psi_{1}(r) M_{0}^{\prime}+r M_{1}^{\prime}(r)-r^{2} \psi_{1}^{\prime}(r)+2 r M_{0}(r) \psi_{1}^{\prime}(r)\right] \varepsilon+O\left(\varepsilon^{2}\right) .
$$

In the simplest case, the classical sources are absent and the zeroth-order solution reduces to the Schwarzschild line element with $M_{0}(r)=\mathcal{M}$. (Note that $M_{0}(r)=\mathcal{M}$ and $M_{1}(r)=0$ as $r \rightarrow \infty)$. Although the renormalized stress-energy tensor in the Schwarzschild spacetime has been calculated by Frolov and Zel'nikov 29] in the present calculations we have used general formulas of Refs. 27 and 28. After some algebra, for the quantized massive scalar field $(s=0)$ in a large mass limit with arbitrary curvature coupling, one has

$$
M^{(0)}(r)=\mathcal{M}-\frac{\mathcal{M}^{2}}{15 \pi m^{2} r^{6}}\left[(11 \mathcal{M}-6 r) \eta+\frac{19}{56} r-\frac{313}{504} \mathcal{M}\right]
$$

and

$$
\psi^{(0)}(r)=\frac{\mathcal{M}^{2}}{\pi m^{2} r^{6}}\left(\frac{7}{15} \eta-\frac{13}{504}\right) .
$$

Here $m$ is a mass of the field whereas $\eta=\xi-1 / 6$ is the numerical parameter and its two particularly appealing values are $\xi=0$ for the minimal and $\xi=1 / 6$ for the conformal coupling. Similarly, for the quantized massive spinor the first-order solution is simply given by

$$
\begin{gathered}
M^{(1 / 2)}(r)=\mathcal{M}-\frac{\mathcal{M}^{2}}{140 \pi m^{2} r^{6}}\left(3 r-\frac{149}{27} \mathcal{M}\right), \\
\psi^{(1 / 2)}(r)=-\frac{11 \mathcal{M}^{2}}{420 \pi m^{2} r^{6}}
\end{gathered}
$$

whereas for the quantized massive vector field one has

$$
\begin{gathered}
M^{(1)}(r)=\mathcal{M}+\frac{\mathcal{M}^{2}}{280 \pi m^{2} r^{6}}\left(37 r-\frac{611}{9} \mathcal{M}\right), \\
\psi^{(1)}(r)=\frac{131 \mathcal{M}^{2}}{840 \pi m^{2} r^{6}} .
\end{gathered}
$$

Eqs.(36), (37) and (39) (44) are, of course, sufficient to calculate the Einstein and Møller energy. On the other hand, combining (38), (34) and (35), for a problem on hand, for $\Delta E$ one has

$$
\Delta E=4 \pi r^{3}\left\langle T_{r}^{r}\right\rangle_{r e n}^{(s)}
$$

It is an interesting result, which clearly shows that the difference between the Møller and Einstein energy is related to the radial pressure of the quantized fields. It can be contrasted with the Radinschi-Yang formula. One can ascribe this behaviour to $\psi(r) \neq 0$, which, in turn, is a consequence of the form of the stress-energy tensor. On the other hand, for the class of the renormalized stress-energy tensors of the quantized fields in a large mass limit in the Bertotti-Robinson (or more generally $A d S_{2} \times S_{2}$ ) geometries one has $\psi(r)=0$. It should be noted that the formula (45) may be used as the useful check of the calculations.

Now, making use of either (38) or (45), one readily obtains

$$
\Delta E^{(0)}=\frac{\mathcal{M}^{2}}{\pi m^{2} r^{6}}\left[\frac{2}{5}(3 \mathcal{M}-2 r) \eta+\frac{1}{24} r-\frac{11}{180} \mathcal{M}\right]=4 \pi r^{3}\left\langle T_{r}^{r}\right\rangle_{r e n}^{(0)}
$$




$$
\Delta E^{(1 / 2)}=\frac{\mathcal{M}^{2}}{\pi m^{2} r^{6}}\left(\frac{r}{20}-\frac{7}{90} \mathcal{M}\right)=4 \pi r^{3}\left\langle T_{r}^{r}\right\rangle_{\text {ren }}^{(1 / 2)}
$$

and

$$
\Delta E^{(1)}=-\frac{\mathcal{M}^{2}}{\pi m^{2} r^{6}}\left(\frac{11 r}{40}-\frac{5}{12} \mathcal{M}\right)=4 \pi r^{3}\left\langle T_{r}^{r}\right\rangle_{\text {ren }}^{(1)}
$$

It should be emphasized once more, that $\left\langle T_{\mu}^{\nu}\right\rangle_{\text {ren }}^{(s)}$ calculated for the zeroth-order solution of the semiclassical Einstein field equations coincides with the stress energy tensor of the massive fields constructed in the Schwarzschild spacetime parametrized by the mass $\mathcal{M}$.

To this end, let us calculate the Einstein energy as the Møller energy can easily be obtained from the definition of $\Delta E^{(s)}$ and (46,48). Inserting the functions $M^{(s)}(r)$ and $\psi^{(s)}(r)$ as given by (39, 44) into (36) (or, equivalently, into (19) and retaining the terms which are linear in $\varepsilon$ ) one obtains

$$
\begin{gathered}
E_{E}^{(0)}=\mathcal{M}+\frac{\mathcal{M}^{2}}{15 \pi m^{2} r^{6}}\left[\frac{59}{252} \mathcal{M}-\frac{19}{56} r+(6 r-4 \mathcal{M}) \eta\right] \\
E_{E}^{(1 / 2)}=\mathcal{M}+\frac{\mathcal{M}^{2}}{\pi m^{2} r^{6}}\left(\frac{5}{378} \mathcal{M}-\frac{3}{140} r\right)
\end{gathered}
$$

and

$$
E_{E}^{(1)}=\mathcal{M}-\frac{\mathcal{M}^{2}}{280 \pi m^{2} r^{6}}\left(\frac{218}{9} \mathcal{M}-37 r\right) .
$$

Finally let us return to the boundary conditions (8) and observe that in this case the solution is parametrized by the exact location of the event horizon, $r_{+}$. The total mass of the system is, therefore, given by

$$
\mathcal{M}=\lim _{r \rightarrow \infty}\left(M_{0(r)}+\varepsilon M_{1}(r)\right),
$$

and it differs from the horizon defined mass, $M_{H}$. As the calculations in this parametrization closely follow the general pattern described above, we shall not display them here.

Our next example is to certain extent related to the previous one: we shall consider the pure gravity action supplemented with the higher curvature terms and analyse the Schwarzschild solutions influenced by all time-reversal-invariant operators of dimension six 33. In doing so we shall assume that the coefficients of the higher curvature part of the action are arbitrary rather than inspired by a particular theory. The action functional has, therefore, the form

$$
S=\int d^{4} x g^{1 / 2} R+S_{1}+S_{2},
$$

where $S_{1}$ is the action functional of the quadratic gravity (that does not influence the problem) and $S_{2}$ is given by

$$
\begin{aligned}
S_{2}= & \int d^{4} x g^{1 / 2}\left(c_{1} R_{\mu \nu \lambda \sigma} R^{\alpha \beta \lambda \sigma} R_{\alpha \beta}^{\mu \nu}+c_{2} R_{\lambda \sigma}^{\mu \nu} R_{\mu \alpha}{ }^{\lambda \beta} R_{\nu \beta}^{\alpha \sigma}\right. \\
& +c_{3} R_{\mu \nu} R^{\mu \alpha \beta \gamma} R_{\alpha \beta \gamma}^{\nu}+c_{4} R R_{\mu \nu \lambda \kappa} R^{\mu \nu \lambda \kappa} \\
& \left.+c_{5} R_{\mu \nu} R_{\lambda \kappa} R^{\mu \lambda \nu \kappa}+c_{6} R_{\mu \nu} R^{\mu \lambda} R_{\lambda}{ }^{\nu}+c_{7} R^{3}+c_{8} R R_{\mu \nu} R^{\mu \nu}\right) .
\end{aligned}
$$

For spherically-symmetric and static systems the perturbative solution is given by (3) with

$$
\psi(r)=-\frac{96 \pi}{r^{6}} \mathcal{M}^{2} \varepsilon\left(18 c_{1}+6 c_{2}+71 c_{3}+28 c_{4}\right)
$$


and

$$
\begin{aligned}
M(r)= & \mathcal{M}-\frac{144 \pi \mathcal{M}^{2}}{r^{5}} \varepsilon\left(12 c_{1}+3 c_{2}+4 c_{3}+16 c_{4}\right) \\
& +\frac{32 \pi \mathcal{M}^{3}}{r^{6}} \varepsilon\left(98 c_{1}+25 c_{2}+33 c_{3}+132 c_{4}\right) .
\end{aligned}
$$

Elementary calculation shows that the difference between Einstein and Møller complexes is given by

$$
\Delta E=-r M_{1}^{\prime}(r)+r^{2} \psi_{1}^{\prime}(r)\left(1-\frac{2 \mathcal{M}}{r}\right) .
$$

Making use of (56) and (57), one arrives at

$$
\begin{aligned}
\Delta E= & \frac{144 \pi \mathcal{M}^{2}}{r^{5}}\left(16 c_{1}+9 c_{2}+8 c_{3}+32 c_{4}\right) \\
& -\frac{192 \pi \mathcal{M}^{3}}{r^{6}}\left(10 c_{1}+11 c_{2}+9 c_{3}+36 c_{4}\right) .
\end{aligned}
$$

It can easily be demonstrated by an independent calculation that

$$
S_{r}^{r}=\Delta E / 8 \pi r^{3},
$$

where

$$
\mathcal{S}^{a b}=\frac{1}{g^{1 / 2}} \frac{\delta S_{2}}{\delta g_{a b}} .
$$

Treating $S^{a b}$ as the effective stress-energy tensor, one concludes that $\Delta E$ is proportional to the radial pressure, as expected.

The results obtained in this paper clearly shows that it is necessary to explain why various pseudotensors are related in a specific way. We suspect that there are other, quite simple but interesting relations which can easily be explained with the aid of the Einstein equations and careful examination of the role played by their source terms. Moreover, it would be interesting to consider other pseudotensors for the whole classes of geometries and relate them with the specific choices of the boundary terms. Finally we observe that the evident direction for further research is generalization of the semi-classical calculations to more complicated settings, such as the Reissner-Nordström spacetime or the geometry of the regular black holes. These issues are under active consideration and the results will be published elsewhere.

\section{Acknowledgments}

The author acknowledges helpful correspondence with Elias Vagenas and his critical reading of the manuscript.

\section{References}

[1] C. C. Chang, J. M. Nester, ans C. M. Chen, Phys. Rev. Lett. 83, 1897 (1999).

[2] J. W. York and D. Brown, Phys. Rev. D 47, 1407 (1993).

[3] C. W. Misner, K. Thorne, and J. A. Wheeler, Gravitation (Freeman, San Francisco, 1973).

[4] See, e.g., A. Trautman, in Gravitation: an Introduction to Current Research, ed. L. Witten (Wiley, New York, 1962), 169. 
[5] L. D. Landau and E. M. Lifshitz, The Classical Theory of Fields, 2nd ed. (Reading, Mass.: Addison-Wesley, 1962).

[6] S. Weinberg, Gravitation and Cosmology (Wiley, New York, 1972).

[7] C. Møller, Ann. Phys. 4, 347 (1958).

[8] P. G. Bergmann and R. Thompson, Phys. Rev. 89, 400 (1953).

[9] A. Papapetrou, Proc. Roy. Irish Acad. A 52, 11 (1948).

[10] K. S. Virbhadra, Phys. Rev. D 41, 1086 (1990).

[11] K. S. Virbhadra, Phys. Rev. D 42, 2919 (1990).

[12] N. Rosen and K. S. Virbhadra, Gen. Relat. Grav. 25, 429 (1993).

[13] K. S. Virbhadra, Pramana 45, 215 (1995).

[14] J. M. Aguirregabiria, A. Chamorro and K. S. Virbhadra, Gen. Relat .Grav. 28, 1393 (1996).

[15] S.S. Xulu, Mod. Phys. Lett. A15, 1511 (2000).

[16] S.S. Xulu, Int. J. Theor. Phys. 46, 2915 (2007).

[17] I. C. Yang and I. Radinschi, Chin. J. Phys. 42, 40 (2004).

[18] E. Vagenas, Mod. Phys. Lett. A 21, 1947 (2006)

[19] E. Ayon-Beato and A. Garcia, Phys. Lett. B464, 25 (1999).

[20] K. A. Bronnikov, Phys. Rev. D63, 044005 (2001).

[21] J. Matyjasek, Phys. Rev. D 70, 047504 (2004).

[22] I. Dyminkova, Gen. Relat. Grav. 24, 235 (1992).

[23] R. Ruffini, astro-ph/9811232.

[24] M. Visser, Phys. Rev. D 46, 2445 (1992).

[25] K. S. Virbhadra, Phys. Rev. D 60, 104041 (1999).

[26] S. S. Xulu, Astrophys. Space Sci. 283, 23 (2003).

[27] J. Matyjasek, Phys. Rev. D 61, 124019 (2000).

[28] J. Matyjasek, Phys. Rev. D 63, 084004 (2001).

[29] V. P. Frolov and A. I. Zel'nikov, Phys. Lett. B 115, 372 (1982).

[30] V. P. Frolov and A. I. Zel'nikov, Phys. Lett. B 123, 197 (1983).

[31] V. P. Frolov and A. I. Zel'nikov, Phys. Rev. D 29, 1057 (1984).

[32] P. R. Anderson, W. A. Hiscock, D. A. Samuel, Phys. Rev. D 51, 4337 (1995).

[33] M. Lu and M. B. Wise, Phys. Rev. D 47, R3095 (1993). 